\title{
Invariant Transformation of Human Time Representations at the Stage of Turning Events
}

\author{
Irina Aleksandrovna Ralnikova ${ }^{1}$, Elena Aleksandrovna Ippolitova ${ }^{1}$, Olga Sergeevna Gurova ${ }^{1}$, Ludmila \\ Denisovna Demina $^{1} \&$ Maksim Aleksandrovich Kostenko ${ }^{1}$ \\ ${ }^{1}$ Altai State University, Russian Federation \\ Correspondence: Irina Aleksandrovna Ralnikova, Lenina pr., 61, Barnaul, 656049, Russian Federation.
}

Received: February 12, 2015 Accepted: March 31, 2015 Online Published: July 30, 2015

doi:10.5539/ass.v11n19p15 URL: http://dx.doi.org/10.5539/ass.v11n19p15

\begin{abstract}
This article is devoted to the actual problem of studying the human representations of the lifetimes at the stage of turning events. Such events as: loss of a marriage partner, diseases associated with malignant neoplasm, deprivation of liberty, disability caused by an injury, and reproductive disorders acted as the turning events. The turning events violate the human self-organization considered as an open psychological system, create a certain bifurcation point for the human being, set the context of uncertainty and stability loss, and thereby determine the potential opportunity of finding new grounds to recover a dynamic equilibrium. The revealed invariant transformations of time representations in the era of reversal of life-violation of time integrity, narrowing of transspective review of life path, actualization of orientation to the past, positive reassessment of the past, loss of value of the present and future time, target and event uncertainty of life prospects, and dispersion of personal responsibility for the future.
\end{abstract}

Keywords: postneonclassic, systemic psychological anthropology, turning events, transformation of time representation

\section{Introduction}

The global changes penetrating all the spheres of human existence reveal the society's need for the innovative thinking, responsible, competitive citizen, who is opened for the future. At the same time, a high rate of changes, increase in the diverse challenges and risks, and raise of life externality keep the very actual problem of human resistance in the changing world. The special field of analysis of the problem discussed by the world psychological science is the time represented in the system of human concept about the life path, as well as the factors and conditions that mediate the diverse reorganization of the system at the critical stages.

The reality of present day clearly demonstrates that the diversity of turning vital events is growing, and the number of people faced with them is expanding. The turning event is able to create the bases for reorganizations (transformations, deformations) by the person of himself/herself, the world picture, and the life picture. The ambiguity, multidirectionality, and unpredictability of such reorganizations and their implications for the human, development, and functioning in the society allows us to emphasize the scientific and practical relevance of the study of the given problem field. The scientific relevance is caused by the aspiration of Psychology to the postneonclassical ideal of rationality, the basic principles of which are the system determination of psyche, anthropologisation, and humanitarisation, this being the reason of new ideas about the Psychological Ontology, which considers the human psyche in the value-semantic fields (Klochko, 2005). The postneonclassical studies are focused on the complex self-developing systems (Prigozhin, 1989, pp. 5-16). The fundamental capacity for self-organization acts as the central element of human psyche (Klochko \& Galazhinsky, 1999). In the present context a human can be comprehended as an open psychological system that generates new psychological formations and relies on them in its self-motion (Klochko, 2005). The postneonclassical ideal of rationality creates bases that allow the psychological science to undertake a new approach to the comprehension of a person's life time, represented in his experience and transformation of representations about the past, present and future in case of reversal of life. The practical relevance is expressed in the development of effective technologies of providing the counseling and psychotherapeutic assistance to the person in experiencing the turning event, caused a failure in his self-organization as the open psychological system, and experiencing the restructuring of axiological coordinates of life world and a scene of life (past, present and future) (Ralnikova, 
2012)

\section{Methods}

In the empirical study of transformations of time representations in the era of life turning-point 395 persons participated: 195 of them were included in the main group (respondents, who experience turning events), among whom there are 70 men and women aged 28-60 with malignant neoplasm at different stages of the disease; 32 men and women aged 60-70, widowed over a year ago; 30 men and women aged 30-55, acquired a disability of second and third groups caused by an injury; 25 men aged 20-27, sentenced to imprisonment and are serving sentences; 40 married women aged 23-35, specially registered due to the reproductive dysfunction. The contrast group was formed for each of the main groups. In total 200 respondents were included in the contrast groups.

The basic method of collecting empirical data consisted of: psycho-diagnostic testing (Time cyclic test (Golovakha \& Kronik, 2008), Time perspective test (Syrtsova et al., 2007, pp. 85-106), Scale of time experience (Golovakha \& Kronik, 2008). Time cyclic test was used in order to identify the subjective importance of temporal modes in the holistic view of life path, and their relatedness to each other. Time perspective test was used in order to determine the temporal orientations of respondents (positive past, negative past, fatalistic present, hedonistic present, and future). The method of Scale of time experience was used to diagnose an emotional attitude to the past, present, and future.

The methods of mathematical and statistical data processing are represented by the procedures for calculating the difference criterion of the averages for independent samples (parametric Student T-test and nonparametric Mann-Whitney U-test), and dispersion analysis, performed using the statistical computing package "SPSS" 17.0.

\section{Results}

\subsection{Time Given in the Experience, from the Standpoints of Classical Ideal of the Rationality}

The historical and psychological analysis of temporal problematic in Psychology shows us the presence of problem field, associated with the study of representations about the life path and psychological time, as well as the transformations and deformations of such representations due to life events that means the turning for the person. Adherence to the methodological position, implying a review of scientific development, including Psychology as a logical shift of paradigms (classical, non-classical, postneonclassical (Znakov, 2008, pp. 69-77), allows you to track the formation of time problem and discover the inner tendency of its development.

During the period of classical ideal of the rationality, the time as a factor of human psyche has acquired the status of an independent scientific problem. By focusing on the idea of understanding the objective natural laws, the attention to the determinism problem, searching for causal relationships using, mostly, the natural science methods (Znokov, 2008, pp. 69-77), the specificity of time of psychic processes as a direct, situational experience has been designated within the classical paradigm (Golovakha \& Kronik, 2008). The attention of scientists was focused on the specifics of direct perception and experience of short time intervals, which were incommensurable with the duration of life in general and subjective time dynamics, by the man (Fraisse, 1957), and its causes (Ornstein, 1970). The important achievement of psychological science at this stage was the belief that the psychological time has its own specifics in comparison with the physical time (Golovakha \& Kronik, 2008).

\subsection{The Problem of Human Time Experience in the Context of Non-Classical Ideal of the Rationality}

At the stage of non-classical ideal of the rationality postulated the multidimensionality, diversity, and heterogeneity of the world the particular importance is attached to the subjectivity of the observer (Znakov, 2008, pp. 69-77). The concepts based on the historical and biographical approaches to studying the human psychology, and regularities of its functioning over time are arising (Golovakha \& Kronik, 2008).

Highlighting the subjective along with the objective, in this field of scientific investigation Psychology falls into the already known "dichotomy of internal and external", and this is reflected in the fact of occurrence of two scientific approaches to the study of human time-evolutionary and genetic, and subjective. The first one reduces the individual time to the psychological changes of the man in the "objective and biographical" time. Here, the time acts as the objective condition of life, independent of the personality, and is increasingly seen in retrospect, as the past time, lived through, the life path (Ananev, 1968; Buhler и Massarik, 1968). The second one represents the opposite tendency-the reduction of human time to the sphere of subjective, the features of experience and time awareness are studied (Cottle, 1976; Nuttin, 1964, pp. 60-82; Rubinstein, 1997). S.L. Rubinstein believed that the subjective time is a real time of psychic processes, states and properties of the personality, in which they are functioning and developing based on the objective temporal relations between the life events of various scales reflected in the direct experience and evaluative comprehension. The subjective of time experiences does 
not mean their illusory nature, this is a relative lifetime of the system-human that quite objectively reflects his life plan (Rubinstein, 1997)

Within the boundaries of discussed problematic issues we placed an emphasis on the causal determination which postulated the division of all things into two realities (objective and subjective) and anchored the primacy of the first reality over the second reality in the classical paradigm, and the primacy of the second reality over the first reality in the non-classical paradigm. Along with that, the need to integrate the objective and subjective aspects of human time is designated in the scientific field (Abulkhanova-Slavskaya, 1991). As an attempt to integrate such an approach was realized through introducing the category of personal time, which is considered as a complex developing holistic systematic formation, and is a special psycho-temporal organization of one's consciousness and self-awareness, behavior and activities by the adult personality (Kovalev, 1991, pp. 5-15). The approach to the personal time removes the restrictions of evolutionary and genetic and subjective approaches and is able to link the objective time requirements and subjective processes of time experience that mediate and regulate the practical organization of time (Golovakha \& Kronik, 2008). At the same time, the non-classical ideal of rationality cannot overcome the dichotomy between the subjective and objective (Klochko, 2005).

\subsection{Temporary Problematic in the Prism of Postneonclassical Ideal of the Rationality}

At the present stage of scientific knowledge development, the formation of tendency of the internal and external convergence is noted (Prigozhin, 1989, pp. 5-16). Thereby creating a zone of paradigms overlapping (Klochko, 2005) that gives the rise to the formation of new postneonclassical ideal of the rationality. The postneonclassical thinking is characterized by the growth of scientists' reflection on the value and meaning categories of human being. "'In the acts of interaction the human gets the opportunity to reflect not only the object, but also himself in it as a personality, which implementing his activity-essence, his needs and goals, and his opportunities through that act..." (Klochko, 2005, p. 90). "The interaction, having the generating effect, makes the sense that is for human even the form of actual experienced "here and now" (the presence), and as a signal of compliance, appearance of outside "friend" in the space, which has not yet been assimilated, but assumes the future" (Klochko, 2005, p. 37). The postneonclassical ideal of rationality introduces the value-meaning categories into the study of psychological time, contributes to solving the integration issue of the internal (subjective) and external (objective) time. In this context, it becomes possible to comprehend the time as a new formation generated by the human. In the interaction between the subject and object a qualitatively new reality not reducible to any subjective or objective is born-supersensible and systematic that has the status of "transition layer" between the objective and subjective realities, which received the designation "man's world" (Klochko, 2005).

\subsection{Turning Events in the Context of the Idea of Systemic Determination}

The analysis of scientific and psychological ideas showed that the category of life events is broad enough in its content, it includes a variety of views, starting from the definition of their formal status as the simplest element of life path to the designation of transforming effect on the human and his self-fulfillment in life. In the context of understanding the human is considered as a self-organizing system that generates the new psychological formations and relies on them in its self-motion (Klochko, 2005), as one of such new formations the life events, having the meaning of turning for the human, may act.

Usage of the term "turning events of the life path" has its own history, where this term is classified as a type of life events. It is noted that the turning events differ with the critical importance and cause reorientation of the subject with the assimilation of events in the external world to the accommodation of you to the new conditions, violating the continuity of existence (Nurkova \& Vasilevskaya, 2003, pp. 93-105). At the same time, the events, implying the transitional periods in a human life, have the status of critical due to their direct connection with the significant changes in the lifestyle (Lazebnaya, 1988, pp. 24-25). The critical life situations are characterized by the inability to live, realize the internal needs of the life (motives, aspirations, values, and others). Such situations cannot be solved by the processes of subject and practical and cognitive activities, and can be solved through the process of their experience, i.e. the special activities for the restructuring of psychological world, aimed at establishing the correspondence between the consciousness and being in order to increase the life meaningfulness (Vasilyuk, 1984).

The analysis of presented points of view shows that the terms provided by different authors to describe the life event, which is granted with the special subjective value, such as turning, and critical, are close in their semantic senses. The commonality of understanding lies in assigning the following characteristics to such kind of events: special status in the subjective picture of life path, lock situation, personality changes, and negative emotional background. The special status of event emphasizes its subjective importance to the human in the context of 
individual history. The specificity of event assessment, depending on the perception of and response to it, is caused by features of the personality, its experience, and the nature of situational context (Antsyferova, 1996, pp. 3-15). The lock situation is associated with the difficulties, and sometimes they are irresistible, in the realization of life goals, aspirations, motives, and values by the man. The negative background, accompanying the experience of turning events, is set by the experiences, which includes fear, shame, guilt, resentment, helplessness, despair, anger, loneliness, hopelessness etc.

In the context of idea of the system determination the turning event can be comprehended as an event that requires the global restructuring of a human as an integrated phenomenon, rather than a well-established process of self-development, based on the constantly reproduced contradiction between the image of the world and the lifestyle. Here this contradiction reaches such a critical level, when you need to change both, understanding of the world, and the whole lifestyle. The turning event can be defined as a psychological new formation generated by the system "a human" in the present, which redefines the image of the past and determines the image of the future, thus it rebuilds the life world structure and parameters of its life-implementation. Such kind of event breaks the realization of man's life intentions, is accompanied by the severe emotional experiences, provokes the awareness of impossibility to resolve the contradictions by means of actual knowledge and experience, and leads to changes in the established life-support in the form of system of attitudes, values, motives, goals, strategies, and life styles. The turning event serves as a point of bifurcation-change of the steady state operation of a man, acting as an open psychological system accompanied by a transition from order to chaos. Along with that, it contains the potential for the further human development (Ralnikova, 2012).

\subsection{Kinds of Turning Events}

Based upon the understanding of critical situations (Vasilyuk, 1984) and crisis types (Osipova, 2005), we may determine the kinds of turning vital events, as which were the event-injury, event-loss, event-crisis, and event-frustration. Event-injury-is a kind of turning events related to the experience of extreme situations (military actions, natural disasters, technological disasters, etc.). Event-loss appears as a kind of turning events, having a sense of loss (loss of significant people, work, health, hope, etc.). Event-frustration is comprehended as a turning event, which introduces the significant stumbling and insurmountable obstacles in the human life in achieving the goal (illness, old age, rules, standards, laws, etc.). Event-crisis is identified by a situation that the person cannot solve the usual way (personal, professional, age crises, etc.).

\subsection{Results of the Empirical Study of Human Time Representations at the Stage of Turning Events}

The empirical study of temporal components of the human life world allows to scientifically prove the existence of diverse image reconstructions of the past, present, future, and picture of the life as a whole in the case of such kinds of turning events as an event-loss (loss of a marriage partner, diseases associated with the malignant neoplasm, imprisonment) and an event-frustration (disability caused by an injury, reproductive disorders)

The statistically significant differences in data of parameters under the research between the main groups and contrast groups indicates that a man tends to represent the personal past, present and future in the case of turning events in the form of individual conglomerates loosely connected, and sometimes not connected with each other. In the temporal continuum the past is estimated as the most positive and a significant period in comparison with the present and future, and that proves the dominant temporal orientation to the past. In cases of various turning events the invariantly paramount importance of that part of the passed life path, to which gives a positive sense, is in contrast to the multifaceted reflection of life events of the past and their consequences. The respondents, who experience the turning events, focus only on the bright moments of their lives-joyful events, pleasant memories, achieved goals, and useful life experience. In their opinion, the past is characterized by calmness, uneventfulness, harmony, and filled with positive emotions, can be controlled and manageable. The estimates of the past of the respondents of contrast groups are positive mostly, and along with that weren't deprived of various (including negative) estimates. The given time period is related for them with experiences of pleasure, joy, inspiration, correlated with a sense of inner freedom, and absence of external factors that can block the satisfaction of actual needs, well as with the clarity, sorted views of the past, predominance of sense of predictability, structured and controlled events, relatively logical coherence of the inner life, comprehensibility of intentions, explainable of positive and negative emotional reactions.

Along with the convexity of the past in the representation of respondents from the main study groups about time of their lives, the now and future gets lower status. Subjectively, the current life period (period of turning events) has a general tendency to be evaluated as a stressful, unpleasant, monotonous, not always monitorable, associated with a sense of lack of strength, exhaustion, need for a sense of calm, peace of mind, and the desire to find safety and support. For the respondents in the contrast groups the present is full of meaning and positive 
emotions, an optimistic vision and a high degree of intellectual and emotional involvement in current events prevail.

On the stage of experience of turning events, human nature is to look into the future with optimism and hope, represent it as organized, under his control, and capable to give him the calmness and peace of mind. At the same time, the turning events actualize the emotional stress about the future life. In the image of the future there is no clear ideas about the goals, planned and expected events, and the continuity of implemented, relevant and potential events is broken. The respondents in the contrast groups demonstrated us their involvement in thinking about the future and planning the life prospects. Their future is full of meaning. It is represented by certain events, which mainly are positive and relate to the sense of inner freedom, absence of external factors that could block the satisfaction of current needs. In the visions of the future the sense of predictability, structuredness, controllability of events, and also of relatively orderliness of the inner life, comprehensibility of intentions, and explainable of emotional reactions prevails. Image of the future is full of optimism.

\subsection{Invariant Transformations of Time Representations in the Era of Reversal of Life}

The results of studying the time representations allow us to comprehend the turning events as a factor, capable to redefine the image of the past, present, and future and become the basis of diverse transformations of human representations of the lifetime as a whole, and past, present and future, in particular. Violation of time integrity, narrowing of transspective review of life path, actualization of temporal orientation to the past, positive reassessment of the past, loss of value of the present and future, target and event uncertainty of life prospects, and dispersion of personal responsibility for the your own future acted as the invariant transformations of human time representations at the stage of turning events.

Violation of time integrity is manifested in the loose coupling of the past, present and future with each other in the subjective image of life, weakening of continuity of temporary stages of life, and imbalance of temporal orientation. Narrowing of transspective review of life path objectifies itself with the event emptiness of the present and future, their weak senses with impressions, activities, and also the one-sided vision of the past. Actualization of temporal orientation to the past is manifested in the increasing importance of that modus for a man in the era of reversal of life along with the present and future, and also the expressed one-sided assessment of such period as an exclusively positive. The comprehension of such transformations allows to see the function, which gives to its own past. This function is in the acquisition of psychological stability in the present to overcome the challenges introduced by a turning event and its consequences due to the construction of the image of a successful life in the past. The loss of importance of the present and future was manifested in reducing the importance of these modes in the general picture of life path, assessment of events belonging to the specified time periods as insufficiently important. The target and event uncertainty of life prospects lies in the lack of human involvement in the process of thinking about its own future, its scanty senses with expected and planned events, reduction in the importance of target prediction. The dispersion of personal responsibility for the future is manifested in the actualization of position of "observer" for your own life, in contrast to the position of an active subject-its "creator".

In general, the turning events violate the self-organization of human as an open psychological system, which is manifested in the mismatch of ideas about the personal past, present and future, violation of event continuity, inability to plan the realistic future needed, determination of available ways to achieve set goals, exploit opportunities of anticipatory reflection, development of personal responsibility for the results of its activities, poor meaningfulness of life choices, inadequate self-analysis of individual activities in the context of developing a life path.

\section{Discussion}

In psychological science the problem of changing the human perceptions about his own life, personal past, present and future in connection with the life events and situations bearing the turning nature is developed (Zimbardo \& Boyd, 2008). The general tendencies manifested in the faint event intensity, discreteness, negative emotional attitude to the future of people at the crisis stages of life path are described (Khomik \& Kronik, 1998, pp. 93-105). Data on the deformations of temporal orientations due to the experience of turning events are known (Akhmerov, 2008, pp. 139-165).

The scientific study made by us helped to confirm the previously described results, and to establish that at the turning stages of life path the semantic field of actual being diffuses, prerequisites for the value and meaning conflicts are developed, responsibility for the results of activities is decreased in the destructured turning event in the space of vital activity, the man's holistic view of the lifetimes is broken, experiences of hopelessness are exacerbated, event-driven and target emptiness arises, and the interest in the self-construction of one's own life is 
lost.

Along with that, we believe that the invariant transformations of time representations caused by the turning event disturb the human self-organization as an open psychological system, create a certain bifurcation point for the human being, set the context of uncertainty, "loss" of sustainability, and thereby determines the potential opportunity of finding new grounds for the recovery of dynamic equilibrium. It's such a bifurcation point that has a dual nature. On the one hand, this is a crisis, turning point, stress, problem situation. On the other hand-reorganization, re-thinking, finding a way out the prevailing circumstances, and possibility of obtaining the order by the psychological system "a human" at a new level.

Not everyone is able to find a way out of such a complex and uncertain life situation on their own, that is imparting the special importance to the psychological science's solution of problem of development of practice-oriented technologies for providing the psychological assistance to a person, who is in the turning events, development of a set of preventive and psycho-correctional measures aimed at optimization of time representations and designing of life prospects.

\section{Conclusion}

Thus, our scientific study has shown that a meaningful as a complicated open self-organizing unit of the psychological system faces with changes in its functions in response to the turning events, which objectifies themselves in the transformations of time representations. In this paper we made an attempt to address the issue of a person's time represented in the experience by the psychological science in the coordinates of postneonclassical ideal of rationality, and it reflects the modern vector of this science development. From the mentioned positions we comprehended the person's time, turning events and their kinds are defined, invariant transformations of time representations in the era of reversal of life are revealed and described. The conducted scientific study reveals the broad prospects for further study of the time issue in Psychology. Firstly, along with the invariant transformations (universal, not related to the specifics of a particular crucial event, that reveal themselves in all research cases) it remains important to determine the variative transformations (unique, typical for a single crucial event, and not in the case of another) of human time representations, who going through the turning events. Secondly, it is necessary to expand the boundaries of our study, both in terms of variety of turning events and determining the age and gender characteristics of perception and experiencing of events that have a critical nature. Thirdly, it is necessary to forward investigational attention to the cross-cultural differences in the psychological time, features of transformation of presentations of the past, present and future of people from different cultures in the context of turning events. Fourth, it's noteworthy-the problem concerning the behavioral response of the man to the bifurcation situation predetermined by the turning event that was revealed by our study. The possible response assumes a risk shift to the deviant behavior forms, (Ralnikova, 2012), that actualizes another direction for further studies of the aforesaid scientific problem. Fifth, it's necessary to create the scientific developments of technologies for the man's psychological assistance at the stage of experience of turning events in the aspect of time coordinates of his life, including the construction of a future needed and development of life prospects.

\section{References}

Abulkhanova-Slavskaya, K. A. (1991). Life strategies. Moscow: Mysl.

Akhmerov, R. A. (2008). Viability as a psychobiographic characteristic (using the example of HIV-infected). Travel time: studies and reflections (pp. 139-165). Kiev.

Ananev, B. G. (1968). A human as an object of knowledge. Leningrad, L: Publishing house of Leningrad University.

Antsyferova, L. I. (1996). Consciousness and actions of individuals in difficult life situations. Psychological magazine, 12(1), 3-15.

Buhler, C., \& Massarik, F. (1968). The course of human life: A study of goals in the humanistic perspective. New York, NY: Springer.

Cottle, T. J. (1976). Perceiving time: A psychological study of men and women. New York, NY: Wiley.

Fraisse, P. (1957). Psychologie du temps. Paris, P. U. F.

Golovakha, E. I., \& Kronik, A. A. (2008). Psychological time of personality. Moscow: Smysl.

Khomik, V. S., \& Kronik, A. A. (1998). Attitude towards time: psychological problems of early alcoholization and deviant behavior. Issues of Psychology, 1, 93-105. 
Klochko, V. E. (2005). Self-organization in the psychological systems: Problems of formation of the mental space of personality (introduction to the transspective analysis). Tomsk: Tomsk State University.

Klochko, V. E., \& Galazhinsky, E. V. (1999). Personal self-fulfillment: Systemic approach. Tomsk: Tomsk State University.

Kovalev, V. I. (1991). Personal time and life path of the personality. Psychology of Personality and of a person's life time (pp. 5-15). Chernovtsy.

Lazebnaya, E. O. (n. d.). Consequences of psychological trauma as a factor of society traumatization. Post-traumatic and post-war stress. Rehabilitation and social adaptation problems of participants of emergency situations: Interdisciplinary approach (pp. 24-25). Perm.

Nurkova, V. V., \& Vassilevskaya, K. N. (2003). Autobiographical memory in difficult life situation: new phenomena. Issues of Psychology, 5, 93-105.

Nuttin, J. R. (1964). The future time perspective in human motivation and learning. Acta Psychologica, 23, 60-82. http://dx.doi.org/10.1016/0001-6918(64)90075-7

Ornstein, R. E. (1969). On the experience of time. Harmondsworth: Penguin.

Osipova, A. A. (2005). Handbook of Psychologist in crisis situations. Rostov-on-Don: Phoenix.

Prigozhin, I. R. (1989). Rediscovery of time. Issues of Philosophy, 8, 5-16.

Ralnikova, I. A. (2012). Restructuring of the human life prospects system at the stage of turning events. Barnaul: Publishing house of the Altai State University.

Rubinstein, S. L. (1997). A human and the world. Moscow: Science.

Syrtsova, A., Sokolova, E. G., \& Mitina, O. V. (2007). Methodology by F. Zimbardo for the temporary perspective. Psychological diagnostics, 1, 85-106.

Vasilyuk, F. E. (1984). Psychology of experience (Analysis of overcoming the critical situations). Moscow: Moscow University Press.

Zimbardo, P. G., \& Boyd, J. N. (2008). The time paradox: The new psychology of time that will change your life. New York, NY: Free Press.

Znakov, V. V. (2008). Psychology of human being-one of the directions of psychology subject development. Psychological magazine, 29(2), 69-77.

\section{Copyrights}

Copyright for this article is retained by the author(s), with first publication rights granted to the journal.

This is an open-access article distributed under the terms and conditions of the Creative Commons Attribution license (http://creativecommons.org/licenses/by/3.0/). 